\title{
Sphingosine-1-phosphate ameliorates the cardiac hypertrophic response through inhibiting the activity of histone deacetylase-2
}

\author{
HUI YAN, SHAOWEI YI, HANG ZHUANG, LUJIN WU, DAO WEN WANG and JIANGANG JIANG \\ Department of Internal Medicine and Institute of Hypertension, Tongji Hospital, Tongji Medical College, \\ Huazhong University of Science and Technology, Wuhan, Hubei 430030, P.R. China
}

Received June 21, 2016; Accepted November 29, 2017

DOI: $10.3892 /$ ijmm.2017.3325

\begin{abstract}
Inhibition of histone deacetylase-2 (HDAC2), which is a prohypertrophic factor in the heart, can functionally attenuate cardiac hypertrophy. The present study aimed to investigate whether sphingosine-1-phosphate (S1P), which has recently been reported to suppress HDAC2 activity, could ameliorate the cardiac hypertrophic response and improve cardiac function in mice with transverse aortic constriction (TAC), as well as to determine the underlying mechanisms. Briefly, 8-week-old male C57BL/6 mice were randomly divided into sham, TAC and TAC + S1P groups; the results indicated that S1P treatment attenuated TAC-induced cardiac dysfunction. In addition, heart size and the expression levels of fetal cardiac genes were reduced in the TAC $+\mathrm{S} 1 \mathrm{P}$ group compared with in the TAC group. Furthermore, in cultured H9c2 cells exposed to phenylephrine, S1P was revealed to decrease cardiomyocyte size and the exaggerated expression of fetal cardiac genes. The present study also demonstrated that S1P had no effect on HDAC2 expression, but it did suppress its activity and increase acetylation of histone $\mathrm{H} 3$ in vivo and in vitro. Krüppel-like factor 4 (KLF4) is an antihypertrophic transcriptional regulator, which mediates HDAC inhibitor-induced prevention of cardiac hypertrophy; in the present study, KLF4 was upregulated by S1P. Finally, the results indicated that S1P receptor 2 (S1PR2) may be involved in the antihypertrophic effects, whereas the suppressive effects of S1P on HDAC2 activity were independent of S1PR2. In conclusion, the present study demonstrated that S1P treatment may ameliorate the cardiac hypertrophic response, which may
\end{abstract}

Correspondence to: Dr Jiangang Jiang or Dr Dao Wen Wang, Department of Internal Medicine and Institute of Hypertension, Tongji Hospital, Tongji Medical College, Huazhong University of Science and Technology, 1095 Jiefang Avenue, Wuhan, Hubei 430030, P.R. China

E-mail: jiangjg618@hotmail.com

E-mail: dwwang@tjh.tjmu.edu.cn

Abbreviations: H3K9-Ac, histone H3 with lysine 9 acetylation; S1PR, sphingosine-1-phosphate receptor; siR, small interfering RNA-S1PR

Key words: sphingosine-1-phosphate, histone deacetylase-2, cardiac hypertrophy, Krüppel-like factor 4, sphingosine-1-phosphate receptor be partly mediated by the suppression of HDAC2 activity and the upregulation of KLF4; it was suggested that S1PR2 may also be involved. Therefore, S1P may be considered a potential therapy for the treatment of heart diseases caused by cardiac hypertrophy.

\section{Introduction}

Cardiac hypertrophy refers to a complex cardiac remodeling process that is induced by various stressors, including hypertension, valve stenosis or regurgitation, and myocardial infarction, thus resulting in an increase in myocardial cell size and heart weight (HW), and ultimately cardiac dysfunction and heart failure $(1,2)$. During this process, the arrested fetal genes that are associated with hypertrophy, including natriuretic peptide type A $(N p p a)$, natriuretic peptide type B $(N p p b)$ and myosin heavy polypeptide $7(M y h 7)$, are reactivated (3). Previous studies have reported that histone deacetylase-2 (HDAC2) serves an important role in cardiac hypertrophy (4,5). HDACs are post-translational modifying enzymes, which can modify the structure of chromosomes and regulate gene expression (6). HDAC2 belongs to class I HDACs and it has previously been demonstrated that inhibiting HDAC2 activity can suppress the progression of cardiac hypertrophy $(7,8)$. Recent progress towards understanding the underlying mechanisms has revealed that the transcriptional regulator Krüppel-like factor 4 (KLF4) mediates HDAC inhibitor-induced prevention of cardiac hypertrophy (9).

Sphingosine-1-phosphate (S1P) is a lysophospholipid mediator that circulates in the blood, which has been reported to be able to inhibit HDAC2 activity, and has been proposed to protect against numerous cardiovascular disorders, including coronary artery disease, atherosclerosis, myocardial infarction and heart failure $(10,11)$. The majority of known S1P effects are based on three specific G protein-coupled receptors (GPCRs), termed S1P receptor (R)1-S1PR3, which are expressed in the cardiovascular system (12). Nevertheless, whether S1P can function through inhibiting HDAC2 in the heart is unknown.

As aforementioned, S1P exerts cardioprotective effects and can suppress HDAC2 activity, which is closely involved in cardiac hypertrophy. However, whether S1P can alleviate cardiac hypertrophy and whether S1PRs participate in it has not been elucidated. Therefore, to further explore the role of S1P in the heart, the present study investigated the effects of $\mathrm{S} 1 \mathrm{P}$ in vivo and in vitro. In vivo experiments were performed on mice under transverse aortic constriction (TAC), which 
were treated with or without S1P. In vitro experiments were performed with $\mathrm{H} 9 \mathrm{c} 2$ cells to explore the effects and mechanisms of S1P.

\section{Materials and methods}

Reagents and antibodies. S1P was purchased from Cayman Chemical Company (Ann Arbor, MI, USA). Phenylephrine (PE) and fluorescein isothiocyanate (FITC)-conjugated wheat germ agglutinin were obtained from Sigma-Aldrich (Merck KGaA, Darmstadt, Germany). Nuclear and Cytoplasmic Protein Extraction kit and DAPI were purchased from Wuhan Boster Biological Technology, Ltd. (Wuhan, China). Cell lysis buffer for western blotting and immunoprecipitation, and actin-Tracker Green were purchased from Beyotime Institute of Biotechnology (Shanghai, China). HDAC2 Fluorimetric Drug Discovery kit was purchased from Enzo Life Sciences, Inc. (Farmingdale, NY, USA). Fetal bovine serum (FBS) and Dulbecco's modified Eagle's medium (DMEM) for cell culture were obtained from Gibco (Thermo Fisher Scientific, Inc., Waltham, MA, USA). Small interfering (si)RNAs were purchased from Guangzhou RiboBio Co., Ltd.(Guangzhou, China). Mega Tran1.0 was purchased from OriGene Technologies, Inc. (Rockville, MD, USA). Antibodies against atrial natriuretic peptide (ANP; sc-20158), brain natriuretic peptide (BNP; sc-271185), S1PR2 (sc-25491) and GAPDH (sc-32233) were from Santa Cruz Biotechnology, Inc. (Dallas, TX, USA). Antibodies against $\beta$-myosin heavy chain ( $\beta$-MHC; 22280-1-AP), HDAC2 (12922-3-AP) and histone H3 (17168-1AP) were obtained from ProteinTech Group, Inc. (Chicago, IL, USA). An antibody against KLF4 (BA3453) was purchased from Wuhan Boster Biological Technology, Ltd. Antibodies against histone H3 with lysine 9 acetylation (H3K9-Ac; ab32129), S1PR1 (ab125074) and S1PR3 (ab108370) were obtained from Abcam (Cambridge, MA, USA).

Animals and animal treatment. Male C57BL/6 mice (age, 8 weeks; weight, 23-26 g; n=24) used in the present study were obtained from the Experimental Animal Center of Changsha (Changsha, China). The present study was approved by the Institutional Animal Research Committee of Tongji Medical College (Wuhan, China). All animal experimental protocols complied with the Guide for the Care and Use of Laboratory Animals published by the United States National Institutes of Health (13). Mice were housed at the Animal Experimental Center of Tongji Hospital (Wuhan, China) at $25^{\circ} \mathrm{C}$, under a 12-h light/dark cycle, and were allowed free access to normal mice chow and water throughout the study period.

Following 1 week acclimation, the mice were randomly divided into various treatment groups. Pressure overload by TAC was used to induce cardiac hypertrophy, as described previously (14). Briefly, a medial skin incision was made from the neck to the upper chest, and the manubrium of the sternum was opened. The transverse aorta between the right innominate artery and left carotid artery was constricted using 7-0 silk suture tied around a 27-gauge needle. Sham surgery was performed without constricting the aorta. A total of 1 week after surgery, TAC-operated mice were randomized into various cohorts, and were intraperitoneally injected with S1P $(6 \mu \mathrm{g} / \mathrm{g} /$ day) or vehicle (saline) for a further 2 weeks (15). The mice were grouped as follows: Sham $(n=8)$, TAC $(n=5)$
Table I. Hemodynamic and echocardiographic results.

\begin{tabular}{lccc}
\hline Variable & Sham & TAC & TAC + S1P \\
\hline Hemodynamics & & & \\
HR (bpm) & $409 \pm 33$ & $375 \pm 9$ & $432 \pm 20$ \\
dp/dt $_{\max }(\mathrm{mmHg} / \mathrm{sec})$ & $5,489 \pm 599$ & $2,356 \pm 390^{\mathrm{a}}$ & $6,330 \pm 1241^{\mathrm{b}}$ \\
$\mathrm{dp}^{\mathrm{d}} \mathrm{dt}_{\min }(\mathrm{mmHg} / \mathrm{sec})$ & $-4,427 \pm 562$ & $-1,620 \pm 369^{\mathrm{a}}$ & $-4,997 \pm 782^{\mathrm{b}}$ \\
Echocardiography & & & \\
EF (\%) & $51.5 \pm 0.9$ & $38.4 \pm 2.5^{\mathrm{a}}$ & $55.0 \pm 2.9^{\mathrm{b}}$ \\
FS (\%) & $26.1 \pm 0.5$ & $18.7 \pm 1.4^{\mathrm{a}}$ & $28.2 \pm 1.8^{\mathrm{b}}$ \\
LVID (d) (mm) & $4.30 \pm 0.13$ & $4.77 \pm 0.10^{\mathrm{a}}$ & $3.81 \pm 0.14^{\mathrm{b}}$ \\
LVID (s) (mm) & $3.22 \pm 0.13$ & $3.85 \pm 0.11^{\mathrm{a}}$ & $2.84 \pm 0.11^{\mathrm{b}}$ \\
\hline
\end{tabular}

Values are presented as the means \pm standard error of the mean; ${ }^{a} \mathrm{P}<0.05$ vs. Sham group; ${ }^{\mathrm{b}} \mathrm{P}<0.05$ vs. TAC group; $\mathrm{n} \geq 5$ /group. $\mathrm{dp} / \mathrm{dt}_{\max }$, maximal slope of systolic pressure increment; $\mathrm{dp} / \mathrm{dt}_{\min }$, minimal slope of diastolic pressure decrement; EF, ejection fraction; FS, fractional shortening; HR, heart rate; LVID (d), left ventricular internal dimension in diastole; LVID (s), left ventricular internal dimension in systole; S1P, sphingosine-1-phosphate; TAC, transverse aortic constriction.

and TAC $+\mathrm{S} 1 \mathrm{P}(\mathrm{n}=6)$ groups. Originally, there were 8 mice in each group; however, following TAC surgery, 3 mice died in the TAC group and 2 died in the TAC + S1P group. This may be the result of the impact of TAC and the specificity of different mice. Hypertrophic responses at the end of the treatment were analyzed by echocardiography, and hemodynamic, histological and biochemical analyses.

Sampling method. Following treatment with S1P for 2 weeks, mice were sacrificed by exsanguination through the carotid artery under pentobarbital $(100 \mathrm{mg} / \mathrm{kg})$ anesthesia; euthanasia was performed in the same way for all of the groups. Subsequently, the whole heart was collected, including the aorta, using ophthalmic scissors to cut along the backbone. The root of the aorta was removed and the heart was separated, weighed and washed with ice-cold saline; one part was stored in liquid nitrogen at $-80^{\circ} \mathrm{C}$ for western blotting and HDAC2 activity assays. The other part of the heart was maintained in $10 \%$ formalin solution for histochemical analysis.

Hemodynamic measurements. Ventricular hemodynamic measurements were performed using a Millar Catheter system (Millar, Inc., Houston, TX, USA) via the right carotid artery under intraperitoneal injection of $100 \mathrm{mg} / \mathrm{kg}$ pentobarbital, as described previously (16). Briefly, a pressure-volume catheter (Millar 1.4F, SPR 835; Millar, Inc.) was inserted into the right carotid artery and advanced into the left ventricle to measure heart rate and instantaneous intraventricular pressure.

Analysis of cardiac function by echocardiography. Cardiac function was assessed by echocardiography, using a high-resolution imaging system equipped with a $30-\mathrm{MHz}$ high frequency scanhead (VisualSonics Vevo770; VisualSonics, Inc., Toronto, ON, Canada) applied to the chest wall. Ventricular dimensions, ejection fraction (EF) and fractional shortening (FS) were examined as described previously (17). 
A

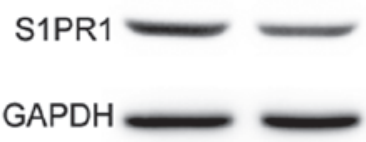

B

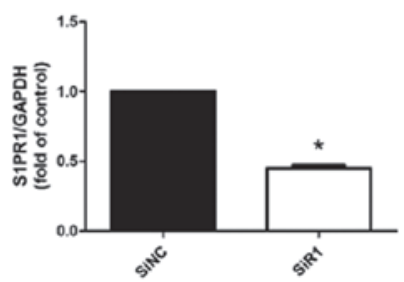

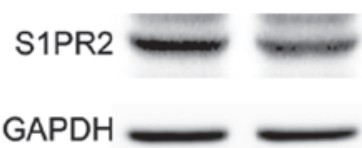

GAPDH -

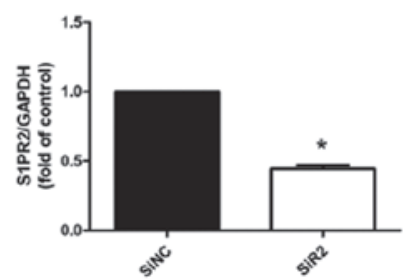

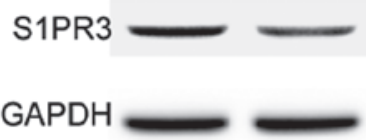

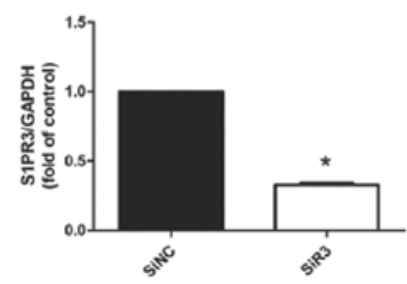

Figure 1. Effects of siRNAs targeting S1PR1-3 in H9c2 cells. (A) Representative immunoblots for S1PR1, S1PR2 and S1PR3 and (B) semi-quantification of S1PRs protein expression in H9c2 cells. Data are presented as the means \pm standard error of the mean ( $\mathrm{n} \geq 3$ for each experiment). ${ }^{*}<0.05$ vs. siNC-transfected cells. S1PR, sphingosine-1-phosphate receptor; siNC, negative control siRNA; siR1-3, siRNA-S1PR1-3; siRNA, small interfering RNA.

Histochemical analysis. Hearts were fixed with $10 \%$ formalin solution for $24 \mathrm{~h}$ at room temperature. They were then embedded in paraffin and sectioned into slices $(5 \mu \mathrm{m})$. To measure the area of cardiomyocytes, heart sections were stained with hematoxylin and eosin (H\&E) and FITC-conjugated wheat germ agglutinin, as previously described (18), and were visualized by light microscopy. Image-Pro Plus Version 6.0 (Media Cybernetics, Inc., Bethesda, MD, USA) was used to measure the area of each cell.

Cell culture and treatments. H9c2 cells, which are a subclone of the original clonal cell line derived from the heart of embryonic BD1X rats, were obtained from American Type Culture Collection (CRL-1446; ATCC, Manassas, VA, USA). The cells were cultured in DMEM supplemented with 10\% FBS and penicillin-streptomycin $(100 \mathrm{IU} / \mathrm{ml})$ in a humidified atmosphere containing $95 \%$ air and $5 \% \mathrm{CO}_{2}$ at $37^{\circ} \mathrm{C}$.

Cells were plated in 6 -well or 12 -well plates at $37^{\circ} \mathrm{C}$, and were treated with $1 \mu \mathrm{M} \mathrm{S1P}$ for $1 \mathrm{~h}$, followed by $100 \mu \mathrm{M}$ PE for $24 \mathrm{~h}$, after which cells were collected. For some experiments, $2 \times 10^{5}$ cells per well were transfected for $24 \mathrm{~h}$ at room temperature with small interfering (si)RNA negative control (siNC) and siRNAs targeting rat S1PR1, S1PR2 and S1PR3 (siR1, siR2 and siR3) using MegaTran 1.0, according to the manufacturer's protocol. The concentration of siRNAs used was $50 \mathrm{nM}$. A total of 24 hours post-transfection, cells were treated with S1P for $1 \mathrm{~h}$ and PE for $24 \mathrm{~h}$.

All in vitro experiments were repeated three times with the same interventions. The siRNA sequences were as follows: siRNA-S1PR1, 5'-CCUCCUUGCUAUCGCCAU UdTdT-3' (sense) and 3'-dTdTGGAGGAACGAUAGCGGUAA-5' (antisense); siRNA-S1PR2, 5'-CCUUGUACGUCCGAAUCU AdTdT-3' (sense) and 3'-dTdTGGAACAUGCAGGCUUAG AU-5' (antisense); siRNA-S1PR3, 5'-GCCACUCUCCAAAGG UCAAdTdT-3' (sense) and 3'-dTdTCGGUGAGAGGUUUC CAGUU-5' (antisense). Successful transfection was confirmed by western blotting (Fig. 1).

Immunocytofluorescence. Cells were initially plated in 12-well plates and were treated as aforementioned. Subsequently, cells were washed with ice-cold PBS, fixed in $4 \%$ paraformalde- hyde for $10 \mathrm{~min}$ and treated with $0.3 \%$ Triton X-100 for $20 \mathrm{~min}$ at room temperature. After blocking with $5 \%$ bovine serum albumin (BSA; Abcam) for $30 \mathrm{~min}$ at room temperature, cells were incubated in actin-Tracker Green at $4^{\circ} \mathrm{C}$ overnight. Subsequently, the cells were stained with DAPI for nuclear detection and were visualized under a Nikon DXM1200 fluorescence microscope (Nikon Corporation, Tokyo, Japan). Image-Pro Plus 6.0 (Media Cybernetics, Inc., Bethesda, MD, USA) was applied to merge images and measure the area of cells.

Immunoprecipitation. Nuclear proteins were extracted using Nuclear and Cytoplasmic Protein Extraction kit according to the manufacturer's protocol, after which they were precleared with protein A/G-agarose beads and incubated overnight with HDAC2 antibody at $4^{\circ} \mathrm{C}$. Subsequently, protein A/G-agarose beads were added and incubated for $2-3 \mathrm{~h}$ at $4^{\circ} \mathrm{C}$ on a rotator. Beads were collected, washed with ice-cold PBS and used for HDAC2 activity assays. Aliquots of agarose-bound immunocomplexes were boiled in SDS-PAGE sample buffer and the released HDAC2 proteins were analyzed by western blot analysis using HDAC2 antibody.

HDAC2 activity assays. HDAC2 activity was tested using a HDAC2 Fluorimetric Drug Discovery kit according to the manufacturer's protocol. Briefly, HDAC2 was isolated by immunoprecipitation and was then subjected to an activity assay. Briefly, samples were incubated with Fluor de Lys ${ }^{\circledR}$-Green Substrate at $37^{\circ} \mathrm{C}$ for $30 \mathrm{~min}$. Fluor de Lys ${ }^{\circledR}$ Developer was then added and the samples were incubated for $15 \mathrm{~min}$ at room temperature. HDAC2 activity levels were expressed as arbitrary fluorescence units and were determined using a Synergy 2 reader (Bio-Tek Instruments, Inc., Winooski, VT, USA) by measuring fluorescence with excitation at $485 \mathrm{~nm}$ and emission at $528 \mathrm{~nm}$.

Western blot analysis. For protein extraction, heart samples and cells were lysed with cell lysis buffer for western blotting and immunoprecipitation. Subsequently, lysates were centrifuged at $12,000 \times \mathrm{g}$ for $20 \mathrm{~min}$ at $4^{\circ} \mathrm{C}$ and the supernatants were used for western blot analysis. The protein concentration was quantified using BCA Protein Assay kit. About $8.5 \mathrm{mg} /$ 
A

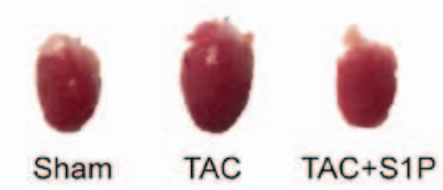

C

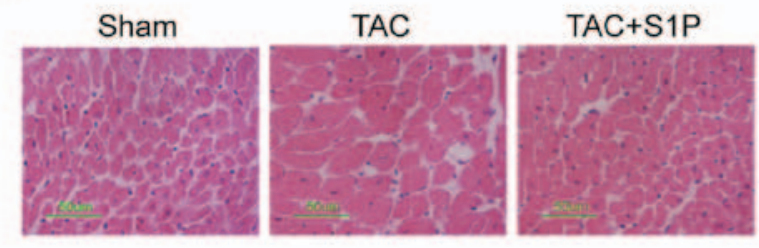

E

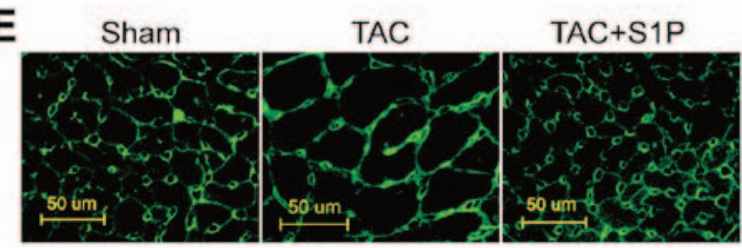

G

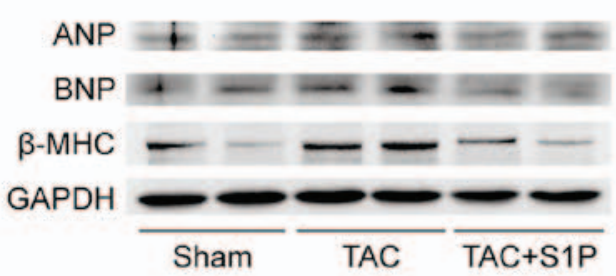

B
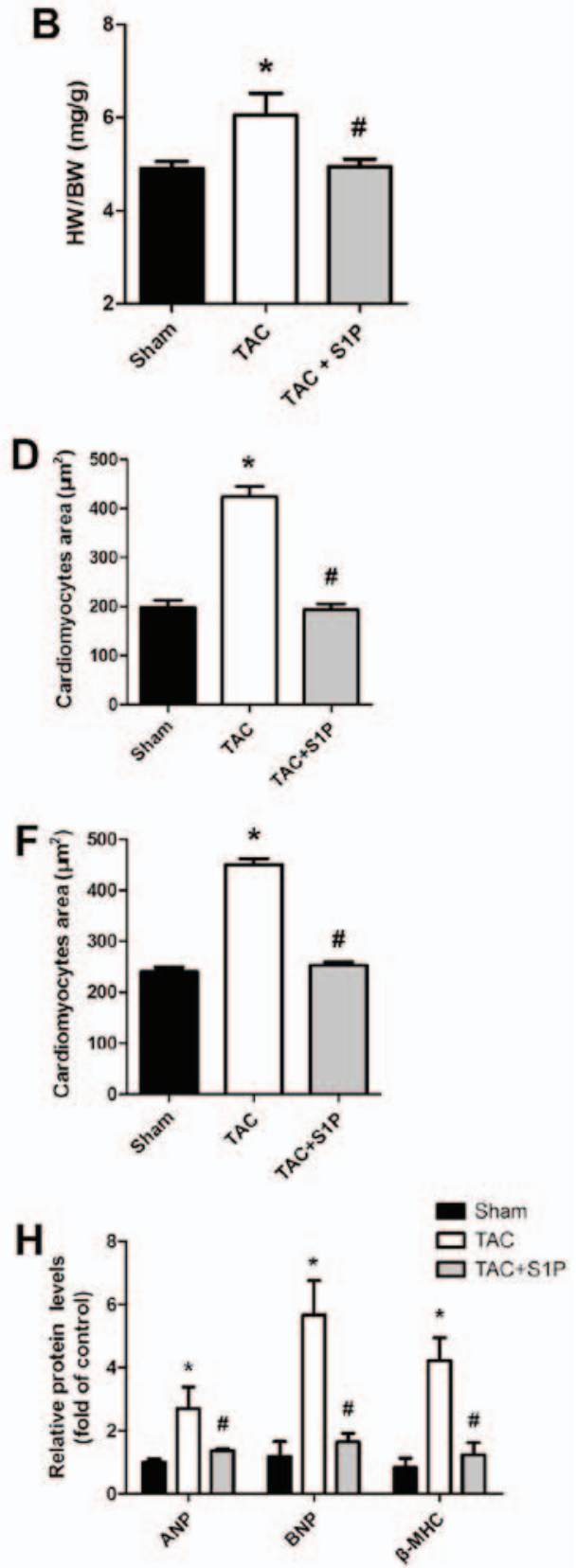

Figure 2. Treatment of mice with S1P prevents the development of TAC-induced cardiac hypertrophy. (A) Representative images of hearts and (B) HW/BW ratios. (C) Histological analysis of cardiomyocytes by hematoxylin and eosin and (D) quantification of cardiomyocyte size. (E) Fluorescein isothiocyanate-conjugated wheat germ agglutinin staining and (F) quantification of cardiomyocyte size. (G) Representative immunoblots for cardiac hypertrophic proteins (ANP, $\mathrm{BNP}$ and $\beta-\mathrm{MHC}$ ) and $(\mathrm{H})$ semi-quantification of cardiac hypertrophic proteins. Data are presented as the means \pm standard error of the mean (n $\geq 5$ for each group). "P<0.05 vs. Sham group; ${ }^{\#} \mathrm{P}<0.05$ vs. TAC group. ANP, atrial natriuretic peptide; BNP, brain natriuretic peptide; $\beta$-MHC, $\beta$-myosin heavy chain; BW, body weight; HW, heart weight; S1P, sphingosine-1-phosphate; TAC, transverse aortic constriction

$\mathrm{ml}$ protein was loaded onto gels. Proteins were subjected to $10 \%$ SDS-PAGE and were transferred onto polyvinylidene fluoride membranes, after which they were incubated at room temperature for $2 \mathrm{~h}$ with blocking solution (5\% BSA), and were then incubated with the indicated antibodies overnight at $4^{\circ} \mathrm{C}$. Rabbit anti-ANP (1:1,000 dilution), rabbit anti-BNP (1:1,000 dilution), rabbit anti- $\beta$-MHC (1:2,000 dilution), mouse anti-GAPDH (1:2,000 dilution), rabbit anti-HDAC2 (1:1,000 dilution), rabbit anti-H3K9-Ac (1:500 dilution), rabbit anti-H3-total (1:2,000 dilution), rabbit anti-KLF4 (1:200 dilution), rabbit anti-S1PR1 (1:5,000 dilution), rabbit anti-S1PR2 (1:1,000 dilution) and rabbit anti-S1PR3 (1:5,000 dilution) were used. Subsequently, these membranes were incubated with a secondary antibody conjugated to horseradish peroxidase (1:5,000 dilution; cat. nos. ab6734 and ab131368; Abcam) at room temperature for $\sim 2 \mathrm{~h}$. Following incubation with each antibody, the membranes were washed five times with Tris-buffered saline-Tween containing $10 \mathrm{mM}$ Tris- $\mathrm{Cl}$ (pH 7.5), $100 \mathrm{mM} \mathrm{NaCl}$, and $0.1 \%$ Tween-20 at room temperature. Immunoreactive bands were examined with enhanced chemiluminescence solution (Thermo Fisher Scientific, Inc.), and were semi-quantified by densitometry and normalized to GAPDH or histone H3 expression. All groups were then normalized to their respective controls. 
A

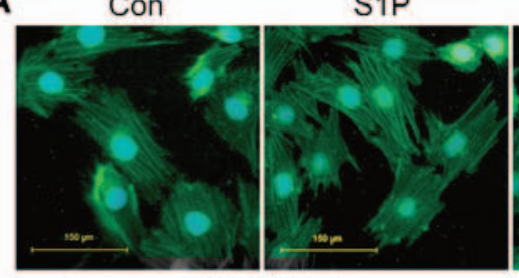

PE

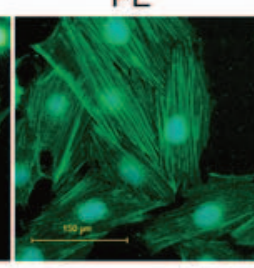

$\mathrm{PE}+\mathrm{S} 1 \mathrm{P}$

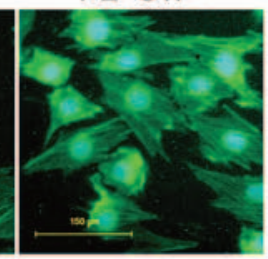

B

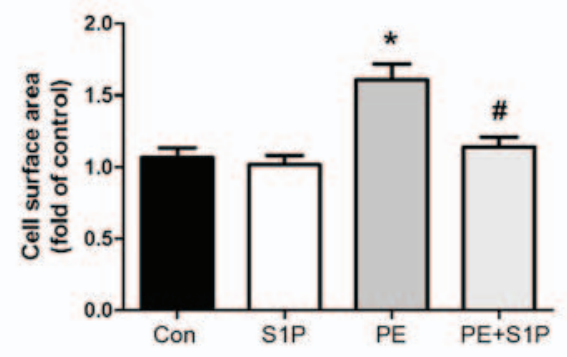

C

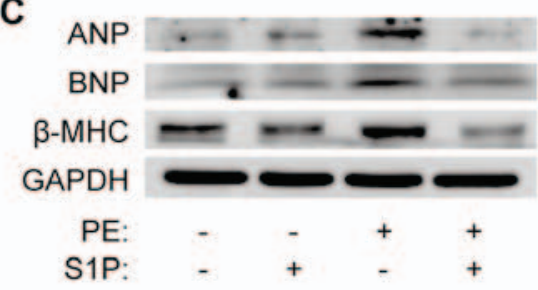

D

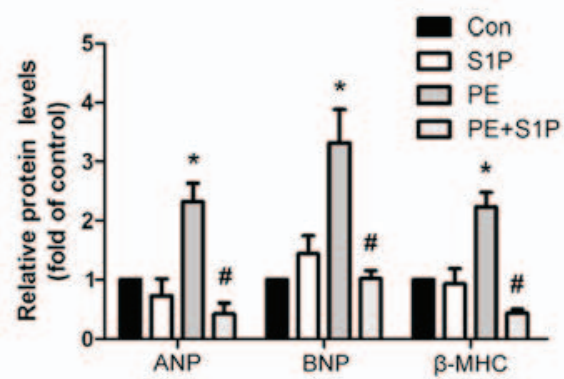

Figure 3. S1P administration inhibits the PE-induced cardiac hypertrophic response in vitro. (A and B) Measurement and quantification of the surface area of H9c2 cells following various treatments (magnification, $\mathrm{x} 400)$. ( $\mathrm{C}$ and D) Representative immunoblots and semi-quantification of cardiac hypertrophy-associated proteins (ANP, BNP and $\beta-\mathrm{MHC}$ ). Data are presented as the means \pm standard error of the mean ( $\mathrm{n} \geq 3$ for each experiment). ${ }^{*} \mathrm{P}<0.05$ vs. Con group; ${ }^{*} \mathrm{P}<0.05$ vs. $\mathrm{PE}$ group. ANP, atrial natriuretic peptide; BNP, brain natriuretic peptide; $\beta$-MHC, $\beta$-myosin heavy chain; Con, control; PE, phenylephrine; S1P, sphingosine-1-phosphate.

Statistical analysis. All continuous data were expressed as the means \pm standard error of the mean. the densitometry was realized using Gel-Pro 32 analyzer and the statistical software used was GraphPad Prism5. Differences between groups were evaluated using unpaired Student's t-test or one-way analysis of variance (ANOVA) and Bonferroni post hoc test. $\mathrm{P}<0.05$ was considered to indicate a statistically significant difference.

\section{Results}

SIP attenuates TAC-induced cardiac dysfunction in mice. To determine the effects of S1P on cardiac performance, heart tissues obtained from the various groups underwent hemodynamic and echocardiographic assessments. The results demonstrated that, compared with in the TAC group, maximal slope of systolic pressure increment and minimal slope of diastolic pressure decrement were consistently increased in the TAC + S1P group; there were no significant alterations in heart rate between the groups (Table I). Enlargement of ventricular chambers at end-systole and end-diastole, and decreased EF and FS were observed following TAC, thus indicating impaired cardiac function. Conversely, compromised cardiac function was significantly improved following S1P treatment (Table I). These data indicated that S1P may ameliorate TAC-induced cardiac function deterioration.

SIP treatment prevents the development of TAC-induced cardiac hypertrophy and reduces the expression of cardiac hypertrophic proteins in mice. To determine the effects of S1P on cardiac hypertrophy, heart weight ( $\mathrm{HW})$ /body weight (BW) ratios and cardiac morphology were compared in mice under various treatment conditions. Heart size and HW/BW ratios were markedly increased in the TAC group; however, in TAC mice treated with S1P, these parameters were reduced to normal levels (Fig. 2A and B). Consistent findings were observed when paraffin-embedded heart sections were stained with H\&E and FITC-conjugated wheat germ agglutinin to evaluate cardiac hypertrophy. The TAC group exhibited larger sizes of cardiomyocytes compared with in the sham group, whereas the TAC + S1P group exhibited similar sizes as the sham group (Fig. 2C-F).

To determine whether TAC induced upregulation of cardiac hypertrophic proteins, western blot analysis was performed to examine the expression levels of ANP, BNP and $\beta$-MHC. The results revealed that TAC increased the expression of these proteins; however, S1P treatment inhibited the expression of these proteins (Fig. $2 \mathrm{G}$ and $\mathrm{H}$ ).

These findings suggested that S1P treatment may prevent the development of TAC-induced cardiac hypertrophy in mice.

SIP administration inhibits the PE-induced cardiac hypertrophic response in cultured $\mathrm{H} 9 \mathrm{c} 2 \mathrm{cells}$. The present study further investigated the effects of S1P on the PE-induced hypertrophic response in $\mathrm{H} 9 \mathrm{c} 2$ cells. Consistent with the in vivo findings, PE $(100 \mu \mathrm{M})$ increased cardiomyocyte size, as detected by cell surface area measurement. Notably, pretreatment with S1P $(1 \mu \mathrm{M})$ restricted this effect (Fig. 3A and B). Furthermore, western blot analysis demonstrated that S1P suppressed the expression of PE-induced markers of cardiac hypertrophy, including ANP, BNP and $\beta$-MHC (Fig. 3C and D). S1P treatment alone had no effect on cardiac hypertrophy compared with in the control group (Fig. 3). These data indicated that S1P could attenuate the cardiac hypertrophic response in vitro.

SIP regulates the cardiac hypertrophic response through inhibiting HDAC2 activity, but not by influencing HDAC2 expression. To test the hypothesis that S1P may regulate the cardiac hypertrophic response by inhibiting HDAC2, the 
A

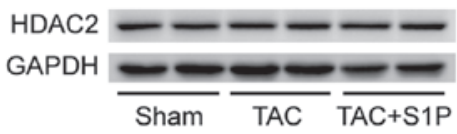

C

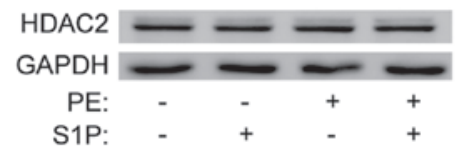

E

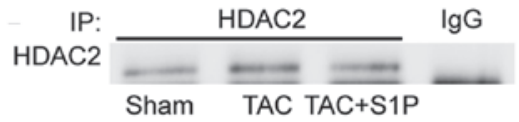

G

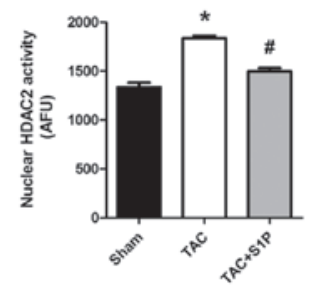

I

H3K9-Ac H3-Total

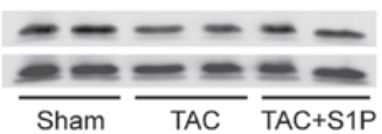

K

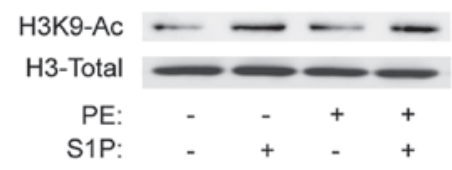

B

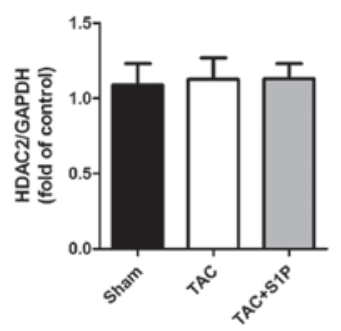

D

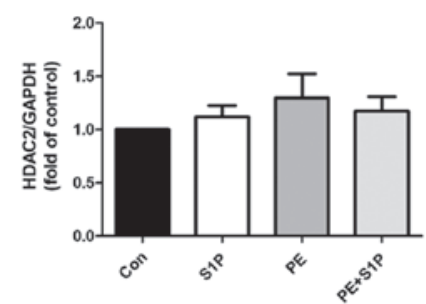

$\mathbf{F}$

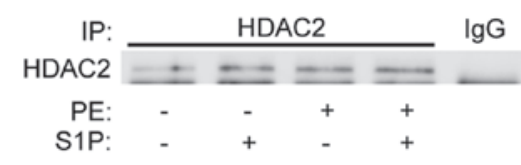

H

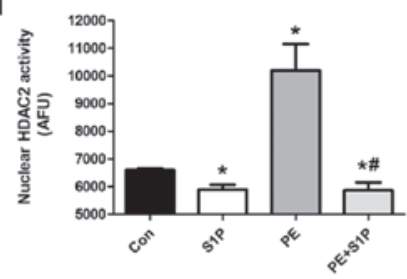

$\mathbf{J}$

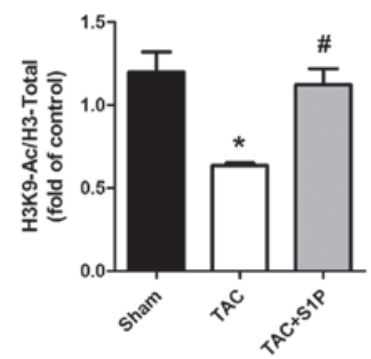

L

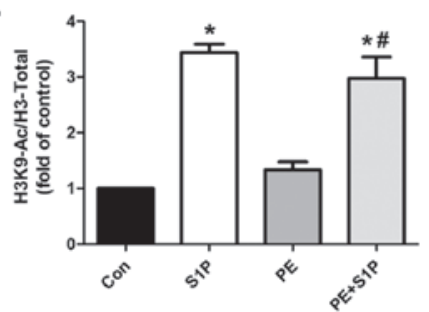

Figure 4. S1P regulates cardiac hypertrophic response through inhibiting HDAC2 activity, but not by affecting HDAC2 expression. (A-D) Representative immunoblots and semi-quantification of HDAC2 expression in heart samples and H9c2 cells. (E and F) IP of HDAC2 in nuclear proteins from various groups. $(\mathrm{G}$ and $\mathrm{H})$ Nuclear HDAC2 activity was determined in duplicate cardiac samples and H9c2 cells. (I-L) H3K9-Ac expression was determined by western blotting with the indicated antibodies. Data are presented as the means \pm standard error of the mean. In vivo experiments, $\mathrm{n} \geq 5$ for each group; ${ }^{*} \mathrm{P}<0.05$ vs. Sham group; ${ }^{\#} \mathrm{P}<0.05$ vs. TAC group; in vitro experiments, $\mathrm{n} \geq 3$ for each experiment; ${ }^{\mathrm{P}}<0.05$ vs. Con group; ${ }^{\mathrm{P}}<0.05$ vs. $\mathrm{PE}$ group. Con, control; $\mathrm{H} 3 \mathrm{~K} 9-\mathrm{Ac}$, histone $\mathrm{H} 3 \mathrm{with}$ lysine 9 acetylation; HDAC2, histone deacetylase-2; IP, immunoprecipitation; PE, phenylephrine; S1P, sphingosine-1-phosphate; TAC, transverse aortic constriction.

expression levels of HDAC2 were detected in heart samples and $\mathrm{H} 9 \mathrm{c} 2$ cells; S1P was revealed to have no effect on HDAC2 expression levels (Fig 4A-D). Therefore, the present study investigated whether S1P could affect HDAC2 activity. HDAC2 was initially extracted from nuclear proteins by immunoprecipitation (Fig. 4E and F) and underwent HDAC2 
B
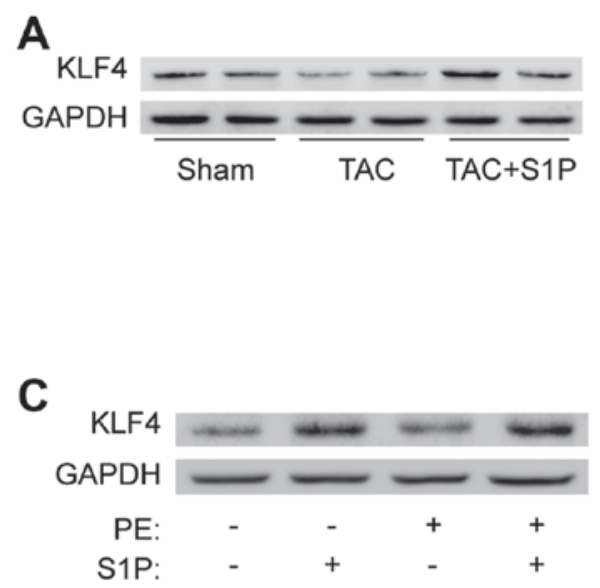

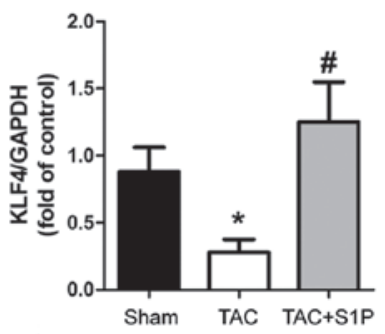

D

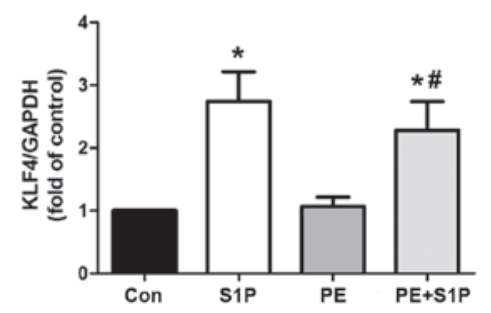

Figure 5. S1P upregulates KLF4, an anti-hypertrophic factor, in vivo and in vitro. (A and B) Representative immunoblots and semi-quantification of KLF4 in heart samples. Data are presented as the means \pm standard error of the mean $\left(\mathrm{n} \geq 5\right.$ for each group). ${ }^{*} \mathrm{P}<0.05$ vs. Sham group; ${ }^{*} \mathrm{P}<0.05$ vs. TAC group. (C and D) Representative immunoblots and semi-quantification of KLF4 in H9c2 cells. Data are presented as the means \pm standard error of the mean ( $\mathrm{n} \geq 3$ for each experiment). "P<0.05 vs. Con group; ${ }^{\#} \mathrm{P}<0.05$ vs. PE group. Con, control; KLF4, Krüppel-like factor 4; PE, phenylephrine; S1P, sphingosine-1-phosphate; TAC, transverse aortic constriction

activity assays. The results indicated that in vivo and in vitro, HDAC2 activity was upregulated under hypertrophic conditions, whereas it was significantly suppressed in groups treated with S1P (Fig. 4G and $\mathrm{H}$ ). To further verify these findings, the expression of H3K9-Ac was detected; treatment with S1P was shown to increase acetylation of H3K9 (Fig. 4I-L).

KLF4, an antihypertrophic factor, is upregulated by S1P in vivo and in vitro. To determine whether S1P, like other HDAC inhibitors, such as trichostatin A, may upregulate KLF4 to prevent the progression of cardiac hypertrophy, the present study measured the expression levels of KLF4 in heart samples. The results demonstrated that KLF4 was significantly upregulated by treatment with S1P compared with in the TAC group (Fig. 5A and B). This increase in KLF4 expression was confirmed in $\mathrm{H} 9 \mathrm{c} 2$ cells (Fig. 5C and D).

S1PR expression is altered following TAC, and S1PR2 may be involved in the antihypertrophic effects of S1P. Since S1PRs have been reported to serve a role in mediating cardioprotection, the present study aimed to determine whether they participate in the antihypertrophic effects of S1P. Initially, the expression levels of S1PR1, 2 and 3 were analyzed, which have been suggested to be expressed in the cardiovascular system of mice $(12,26)$. The results indicated that the expression of all three S1PRs was altered following TAC; S1PR1 and S1PR3 were upregulated, whereas S1PR2 was downregulated, thus suggesting that S1PRs may be involved in cardiac hypertrophy (Fig. 6A and B). Subsequently, S1PR-specific siRNAs were used to investigate whether S1P attenuated cardiac hypertrophic responses through $\mathrm{S} 1 \mathrm{PR}$ in H9c2 cells. The results demonstrated that the representative hypertrophic marker, $\beta$-MHC, was upregulated by PE; however, expression was decreased by S1P and this protective effect was partially suppressed by siR 2 , but not by siR 1 or siR3 (Fig. 6C-H). These findings suggested that S1PR2 may be involved in the antihypertrophic effects of S1P.
Suppressive effects of S1P on HDAC2 activity are independent of S1PR2. Since S1PR2 may be involved in the antihypertrophic effects of S1P, the present study aimed to determine whether inhibition of the effects of S1P on HDAC2 activity was S1PR2-dependent. HDAC2 was extracted from nuclear proteins by immunoprecipitation and its activity was detected following siR2 transfection. The results indicated that S1P significantly inhibited PE-induced upregulation of HDAC2 activity; however, siR2 did not suppress this effect (Fig. 7A). Furthermore, the downstream factor of HDAC2, KLF4, was investigated. The results demonstrated that siR2 did not affect the S1P-induced upregulation of KLF4 (Fig. 7B and C). These findings suggested that the suppressive effects of S1P on HDAC2 activity were independent of S1PR2.

\section{Discussion}

In the present study, a mouse model of TAC-induced cardiac hypertrophy was generated, as well as a PE-induced $\mathrm{H} 9 \mathrm{c} 2$ cardiac hypertrophy cell model. Mice and cells were administered S1P in order to investigate its effects on the cardiac hypertrophic response and the underlying mechanisms. The results indicated that S1P could attenuate the progression of cardiac hypertrophy, and this may be mediated by inhibiting HDAC2 activity, and upregulating KLF4 independently of S1PRs. The results demonstrated that administration of S1P ameliorated TAC-induced cardiac function deterioration, and reduced the hypertrophic response in TAC-treated mice and PE-treated H9c2 cells. Furthermore, the expression levels and activity of HDAC2 were detected; the results suggested that S1P treatment did not affect HDAC2 expression but it did decrease its activity. In addition, the downstream antihypertrophic factor of HDAC2, KLF4, was examined and the results indicated that S1P upregulated KLF4, which may be associated with the suppression of HDAC2 activity. S1PR2 was revealed to be potentially involved in the anti-hypertro- 
A

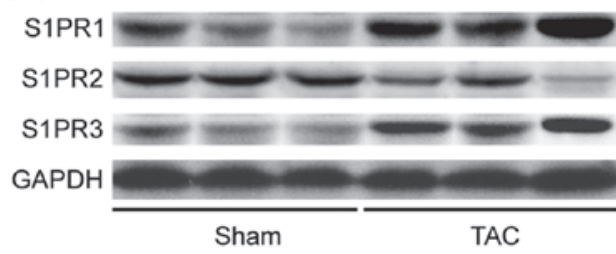

C

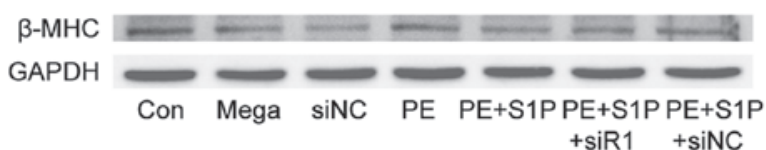

E

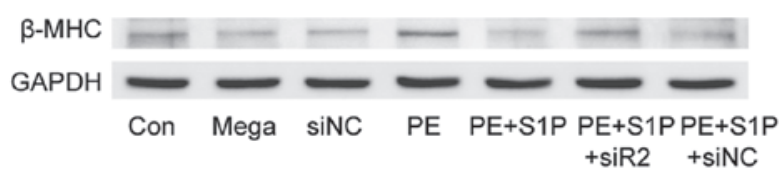

G

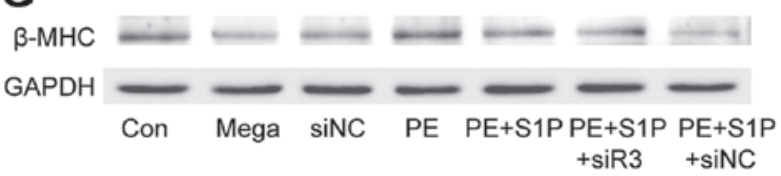

B

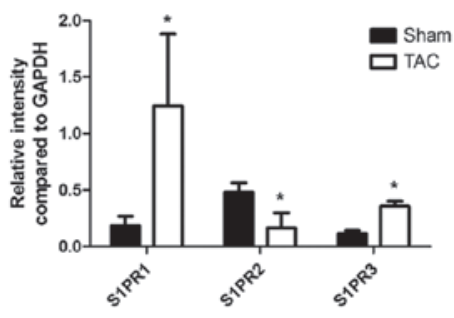

D

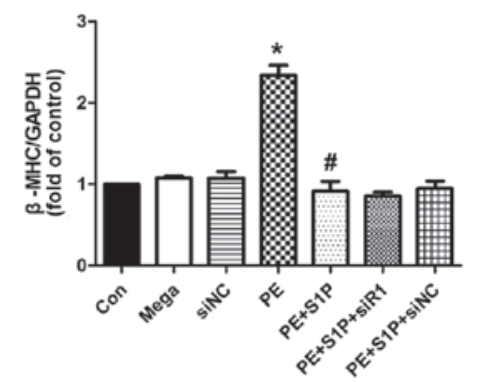

F

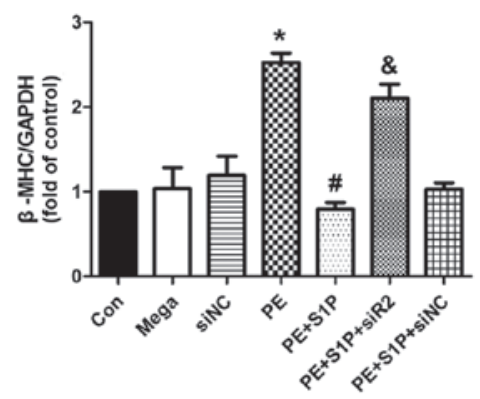

H

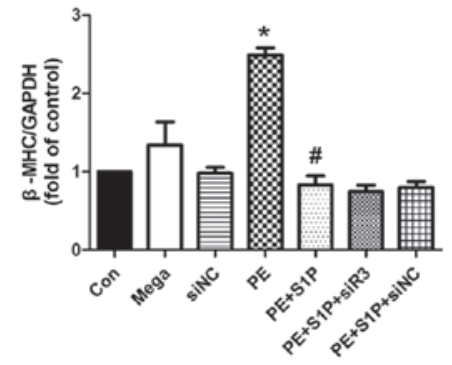

Figure 6. S1PR expression is altered following TAC; S1PR2 may be involved in the antihypertrophic effects of S1P. (A and B) Representative immunoblots and semi-quantification of S1PRs in the hearts of mice in the sham and TAC groups. Data are presented as the means \pm standard error of the mean (n $\geq 5$ for each group). "P $<0.05$ vs. the Sham group. (C-H) Representative immunoblots and semi-quantification of $\beta$-MHC in H9c2 cells following various treatments Data are presented as the means \pm standard error of the mean ( $\mathrm{n} \geq 3$ for each experiment). ${ }^{*} \mathrm{P}<0.05$ vs. Con group; ${ }^{\sharp \mathrm{P}}<0.05$ vs. $\mathrm{PE}$ group; ${ }^{\&} \mathrm{P}<0.05$ vs. $\mathrm{PE}+\mathrm{S} 1 \mathrm{P}$ group. $\beta$-MHC, $\beta$-myosin heavy chain; Con, control; PE, phenylephrine; S1P, sphingosine-1-phosphate; siNC, negative control siRNA; siR1-3, siRNA-S1P receptors 1-3; siRNA, small interfering RNA; TAC, transverse aortic constriction.

phic effects of S1P, whereas S1P functioned independently of it. These findings are summarized in the schematic diagram presented in Fig. 8.

Cardiac hypertrophy is a common pathological characteristic of numerous heart diseases and usually occurs in response to pressure overload, volume overload or myocardial infarction (19). S1P is a lipid mediator formed by metabolism of sphingomyelin, which regulates important functions in cardiac and vascular homeostasis (20). It can increase viability of cardiomyocytes incubated under hypoxic conditions and reduce infarct size in isolated, perfused rat hearts following ischemia/reperfusion $(21,22)$. Furthermore, it participates in the regulation of vascular tone and permeability of vessels $(12,23-25)$. The majority of these effects are mediated by the activation of S1PRs. It has previously been reported that S1P has five specific GPCRs, S1PR1-S1PR5; however, only S1PR1, S1PR2 and S1PR3 are mainly expressed in the heart $(12,26)$. Binding of S1P to each of these receptors activates distinct intracellular signals. A previous study indicated that S1PR signaling is important in heart development, and influences the migration, differentiation and survival of embryonic cardiomyocytes (27). In the present study, the expression levels of S1PRs were markedly altered in hypertrophic hearts; S1PR1 and S1PR3 were upregulated, whereas S1PR2 was downregulated, thus suggesting an association between S1PRs and cardiac hypertrophy.

Hait $e$ t al previously reported that S1P could function independently of S1PRs, through binding HDAC2 and inhibiting its enzymatic activity (11). HDACs are post-translational modifying enzymes that can remove acetyl functional groups 


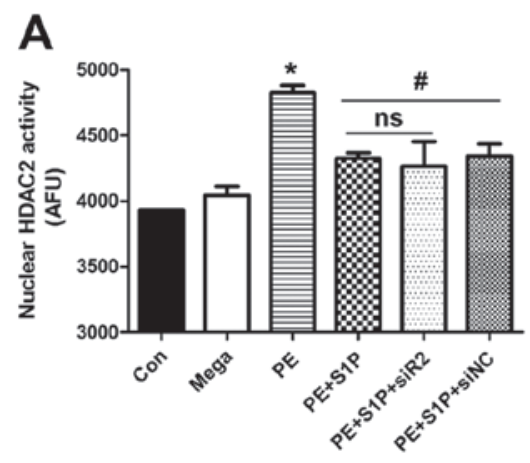

B

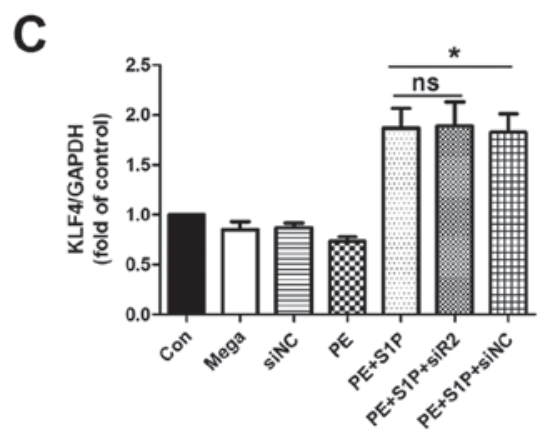

Figure 7. Suppressive effects of S1P on HDAC2 activity are independent of S1PR2. (A) Nuclear HDAC2 activity was determined in H9c2 cells following various treatments. (B and C) Representative immunoblots and semi-quantification of KLF4 expression in H9c2 cells following various treatments. Data are presented as the means \pm standard error of the mean ( $\mathrm{n} \geq 3$ for each experiment). ${ }^{*} \mathrm{P}<0.05$ vs. Con group; ${ }^{\#} \mathrm{P}<0.05$ vs. $\mathrm{PE}$ group; ns, not significant. Con, control; HDAC2, histone deacetylase-2; KLF4, Krüppel-like factor 4; PE, phenylephrine; S1P, sphingosine-1-phosphate; siNC, negative control siRNA; siR2, siRNA-S1PR2; siRNA, small interfering RNA

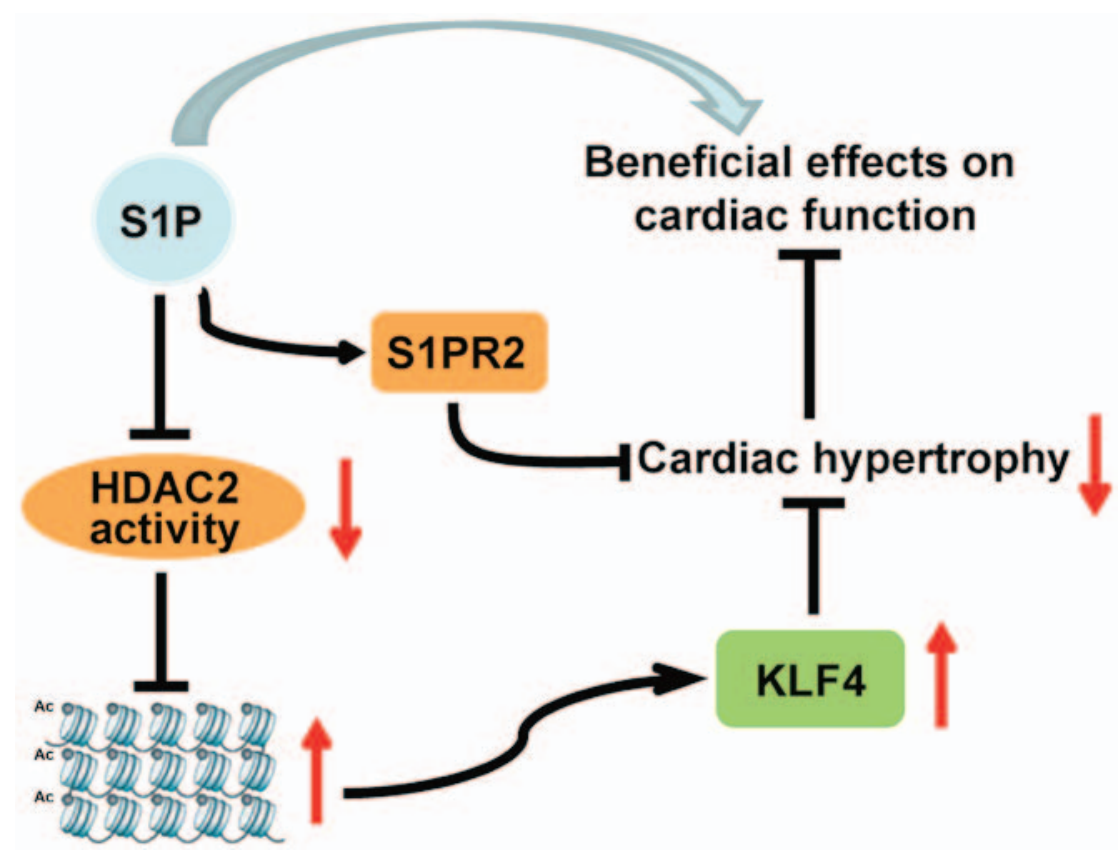

Figure 8. Model of the mechanisms underlying the ameliorative effects of S1P on cardiac hypertrophy. S1P treatment inhibited HDAC2 activity, resulting in increased histone acetylation and upregulation of KLF4, thus ameliorating cardiac hypertrophy. Cardiac hypertrophy is potentially also mediated by S1PR2. These effects may contribute to the improvement of cardiac function. HDAC2, histone deacetylase-2; KLF4, Krüppel-like factor 4; S1P, sphingosine-1-phosphate; S1PR2, S1P receptor 2.

from lysine residues of histone and nonhistone proteins (28). Previous studies regarding HDAC inhibitors have provided evidence to suggest that class I HDACs are prohypertrophic, among which HDAC2 is predominantly activated by hypertrophic stress; activated HDAC2 triggers hypertrophy through inhibiting the signaling cascades of $\operatorname{KLF} 4(6,9)$. Therefore, a selective inhibitor of HDAC2 is considered an effective treatment for cardiac hypertrophy $(7,8)$.

In the present study, the effects of S1P on cardiac hypertrophy were investigated. There are conflicting data regarding 
the role of S1P in cardiac hypertrophy. A previous study performed in neonatal rat cardiomyocytes demonstrated that S1P did not induce hypertrophy, as determined by measuring ANP expression and phenylalanine incorporation, whereas the related sphingolipid, sphingosylphosphorylcholine, was able to induce hypertrophy (29). However, the underlying mechanism remained unclear. Data from another study indicated that S1P induced hypertrophy in neonatal rat cardiomyocytes, as assessed by cell size, cytoskeletal organization, phenylanine incorporation and BNP expression; this hypertrophic response appeared to be mediated by S1PR1 (30). Nevertheless, S1P treatment has not previously been performed under hypertrophic conditions, and it should be recognized that S1P-induced cardiac hypertrophy is less robust and occurs more slowly than the canonical hypertrophic responses elicited by phenylyephrine and endothelin (31).

Since Hait et al reported that S1P could inhibit HDAC2 activity, the effects of S1P have been studied on diseases associated with HDAC2. For example, a recent study demonstrated that S1P increased the ability of muscle cells to use fatty acids as an energy source in mice with Duchenne muscular dystrophy through inhibiting HDAC2 activity and increasing the expression of beneficial muscle genes (32). S1P has also been reported to protect the liver from lipid metabolism dysfunction in mice fed a high fat diet by decreasing HDAC2 activity and upregulating key genes encoding nuclear receptors/enzymes involved in nutrient metabolism (33). The present study demonstrated that S1P was able to prevent cardiac hypertrophy via the suppression of HDAC2 activity; this finding was in agreement with previous reports, which suggested that inhibition of HDAC2 may suppress cardiac hypertrophy $(7,8)$.

KLF4 belongs to a large family of transcription factors named KLFs, which have common structures, including a transcriptional activation/repression domain and three Krüppel-like zinc fingers (34). Previous studies have indicated that KLF4 is a novel regulator of cardiac hypertrophy that is responsible for HDAC inhibitor-induced prevention of cardiac hypertrophy $(9,35,36)$. It was previously revealed that KLF4 bound to the Nppa promoter region from -130 to $\sim-105$ bp downregulates its expression and suppresses cardiac hypertrophy (9). The present study expanded on this established mechanism to determine how S1P regulates cardiac hypertrophy; the results demonstrated that the protective effects of S1P may be mediated by upregulating KLF4.

Notably, cardiac function in the TAC + S1P group was a little better than in the Sham group. We considered that cardiac function would be improved after one week of TAC when cardiac hypertrophy was adapted and compensatory and at that time we treated mice with S1P. Furthermore, the known beneficial effects of S1P on the cardiovascular system may be responsible for this phenomenon. H3K9-Ac and KLF4 were downregulated in mice in the TAC group compared with in the Sham group, whereas PE had no effects on H3K9-Ac and KLF4 expression in H9c2 cells. This may be due to the different in vivo and in vitro environments. In addition, their expression levels were originally low in control H9c2 cells. In cultured H9c2 cells, S1P upregulated H3K9-Ac and KLF4 expression; however, it had no effect on the expression of hypertrophic markers (ANP, BNP and $\beta$-MHC) compared with in the control group. The reason for this may be that the expression levels of hypertrophic markers were originally low under control condition.
In conclusion, the present study indicated that S1P attenuates cardiac hypertrophy by inhibiting HDAC2 activity, thus resulting in the upregulation of KLF4 in a S1PR2-independent manner. These findings may provide important information regarding the potential clinical applications to prevent cardiac hypertrophy. However, whether S1P directly binds to HDAC2 in cardiomyocytes and in mouse models, or whether there are other possible mechanisms underlying S1P-induced inactivation of HDAC2, requires further exploration in future studies, and the role of S1PR2 in hypertrophy should be confirmed in S1PR2-knockout mice.

\section{Acknowledgements}

The authors would like to thank Dr Xingxu Wang for assistance in echocardiography and Xingwei He for assistance in language editing.

\section{References}

1. Eom GH and Kook H: Posttranslational modifications of histone deacetylases: Implications for cardiovascular diseases. Pharmacol Ther 143: 168-180, 2014.

2. Frey N and Olson EN: Cardiac hypertrophy: The good, the bad, and the ugly. Annu Rev Physiol 65: 45-79, 2003.

3. Chang L, Kiriazis H, Gao XM, Du XJ and El-Osta A: Cardiac genes show contextual SWI/SNF interactions with distinguishable gene activities. Epigenetics 6: 760-768, 2011.

4. Eom GH and Kook H: Role of histone deacetylase 2 and its posttranslational modifications in cardiac hypertrophy. BMB Rep 48: 131-138, 2015.

5. Trivedi CM, Luo Y, Yin Z, Zhang M, Zhu W, Wang T, Floss T, Goettlicher M, Noppinger PR, Wurst W, et al: Hdac2 regulates the cardiac hypertrophic response by modulating Gsk3 beta activity. Nat Med 13: 324-331, 2007.

6. Kee HJ and Kook H: Roles and targets of class I and IIa histone deacetylases in cardiac hypertrophy. J Biomed Biotechnol 2011: 928326, 2011.

7. Kee HJ, Sohn IS, Nam KI, Park JE, Qian YR, Yin Z, Ahn Y, Jeong MH, Bang YJ, Kim N, et al: Inhibition of histone deacetylation blocks cardiac hypertrophy induced by angiotensin II infusion and aortic banding. Circulation 113: 51-59, 2006.

8. Kong Y, Tannous P, Lu G, Berenji K, Rothermel BA, Olson EN and Hill JA: Suppression of class I and II histone deacetylases blunts pressure-overload cardiac hypertrophy. Circulation 113: 2579-2588, 2006.

9. Kee HJ and Kook H: Krüppel-like factor 4 mediates histone deacetylase inhibitor-induced prevention of cardiac hypertrophy. J Mol Cell Cardiol 47: 770-780, 2009.

10. Takuwa Y, Okamoto Y, Yoshioka K and Takuwa N: Sphingosine-1-phosphate signaling and biological activities in the cardiovascular system. Biochim Biophys Acta 1781: 483-488, 2008

11. Hait NC, Allegood J, Maceyka M, Strub GM, Harikumar KB, Singh SK, Luo C, Marmorstein R, Kordula T, Milstien S, et al: Regulation of histone acetylation in the nucleus by sphingosine-1-phosphate. Science 325: 1254-1257, 2009.

12. Means CK and Brown JH: Sphingosine-1-phosphate receptor signalling in the heart. Cardiovasc Res 82: 193-200, 2009.

13. National Research Council (US) Committee for the Update of the Guide for the Care and Use of Laboratory Animals. Washington (DC): National Academies Press (US); 2011.

14. Takimoto E, Champion HC, Li M, Belardi D, Ren S, Rodriguez ER, Bedja D, Gabrielson KL, Wang Y and Kass DA: Chronic inhibition of cyclic GMP phosphodiesterase 5A prevents and reverses cardiac hypertrophy. Nat Med 11: 214-222, 2005.

15. Liu W, Zi M, Tsui H, Chowdhury SK, Zeef L, Meng QJ, Travis M, Prehar S, Berry A, Hanley NA, et al: A novel immunomodulator, FTY-720 reverses existing cardiac hypertrophy and fibrosis from pressure overload by targeting NFAT (nuclear factor of activated T-cells) signaling and periostin. Circ Heart Fail 6: 833-844, 2013. 
16. Cingolani $\mathrm{OH}$, Yang XP, Cavasin MA and Carretero OA: Increased systolic performance with diastolic dysfunction in adult spontaneously hypertensive rats. Hypertension 41: 249-254, 2003.

17. Ma H, Gong H, Chen Z, Liang Y, Yuan J, Zhang G, Wu J, Ye Y, Yang C, Nakai A, et al: Association of Stat3 with HSF1 plays a critical role in G-CSF-induced cardio-protection against ischemia/reperfusion injury. J Mol Cell Cardiol 52: 1282-1290, 2012.

18. Dolber PC, Bauman RP, Rembert JC and Greenfield JC Jr: Regional changes in myocyte structure in model of canine right atrial hypertrophy. Am J Physiol 267: H1279-H1287, 1994.

19. Sag CM, Santos CX and Shah AM: Redox regulation of cardiac hypertrophy. J Mol Cell Cardiol 73: 103-111, 2014.

20. Knapp M: Cardioprotective role of sphingosine-1-phosphate. J Physiol Pharmacol 62: 601-607, 2011.

21. Karliner JS, Honbo N, Summers K, Gray MO and Goetzl EJ The lysophospholipids sphingosine-1-phosphate and lysophosphatidic acid enhance survival during hypoxia in neonatal rat cardiac myocytes. J Mol Cell Cardiol 33: 1713-1717, 2001.

22. Lecour S, Smith RM, Woodward B, Opie LH, Rochette L and Sack MN: Identification of a novel role for sphingolipid signaling in TNF alpha and ischemic preconditioning mediated cardioprotection. J Mol Cell Cardiol 34: 509-518, 2002.

23. Alvarez SE, Milstien S and Spiegel S: Autocrine and paracrine roles of sphingosine-1-phosphate. Trends Endocrinol Metab 18 300-307, 2007.

24. Fyrst $\mathrm{H}$ and Saba JD: An update on sphingosine-1-phosphate and other sphingolipid mediators. Nat Chem Biol 6: 489-497, 2010.

25. Strub GM, Maceyka M, Hait NC, Milstien S and Spiegel S: Extracellular andintracellularactions of sphingosine-1-phosphate. Adv Exp Med Biol 688: 141-155, 2010.

26. Spiegel S and Milstien S: Functions of a new family of sphingosine-1-phosphate receptors. Biochim Biophys Acta 1484 107-116, 2000.

27. Wendler CC and Rivkees SA: Sphingosine-1-phosphate inhibits cell migration and endothelial to mesenchymal cell transformation during cardiac development. Dev Biol 291: 264-277, 2006.
28. Seto E and Yoshida M: Erasers of histone acetylation: The histone deacetylase enzymes. Cold Spring Harb Perspect Biol 6: a018713, 2014.

29. Sekiguchi K, Yokoyama T, Kurabayashi M, Okajima F and Nagai R: Sphingosylphosphorylcholine induces a hypertrophic growth response through the mitogen-activated protein kinase signaling cascade in rat neonatal cardiac myocytes. Circ Res 85: 1000-1008, 1999.

30. Robert P, Tsui P, Laville MP, Livi GP, Sarau HM, Bril A and Berrebi-Bertrand I: EDG1 receptor stimulation leads to cardiac hypertrophy in rat neonatal myocytes. J Mol Cell Cardiol 33: 1589-1606, 2001.

31. Wei L: Lysophospholipid signaling in cardiac myocyte hypertrophy. J Mol Cell Cardiol 36: 465-468, 2004.

32. Nguyen-Tran DH, Hait NC, Sperber H, Qi J, Fischer K, Ieronimakis N, Pantoja M, Hays A, Allegood J, Reyes M, et al: Molecular mechanism of sphingosine-1-phosphate action in Duchenne muscular dystrophy. Dis Model Mech 7: 41-54, 2014.

33. Nagahashi M, Takabe K, Liu R, Peng K, Wang X, Wang Y, Hait NC, Wang X, Allegood JC, Yamada A, et al: Conjugated bile acid-activated S1P receptor 2 is a key regulator of sphingosine kinase 2 and hepatic gene expression. Hepatology 61: 1216-1226, 2015.

34. Pearson R, Fleetwood J, Eaton S, Crossley M and Bao S: Krüppel-like transcription factors: A functional family. Int J Biochem Cell Biol 40: 1996-2001, 2008.

35. Yoshida T, Yamashita M, Horimai C and Hayashi M: Kruppel-like factor 4 protein regulates isoproterenol-induced cardiac hypertrophy by modulating myocardin expression and activity. J Biol Chem 289: 26107-26118, 2014.

36. Liao X, Haldar SM, Lu Y, Jeyaraj D, Paruchuri K, Nahori M, Cui Y, Kaestner KH and Jain MK: Krüppel-like factor 4 regulates pressure-induced cardiac hypertrophy. J Mol Cell Cardiol 49: 334-338, 2010 